\title{
Intrinsic electron spin relaxation due to the D'yakonov-Perel' mechanism in monolayer $\mathrm{MoS}_{2}$
}

\author{
L. Wang and M. W. Wu* \\ Hefei National Laboratory for Physical Sciences at Microscale and Department of Physics, \\ University of Science and Technology of China, Hefei, Anhui, 230026, China
}

(Dated: August 15, 2018)

\begin{abstract}
Intrinsic electron spin relaxation due to the D'yakonov-Perel' mechanism is studied in monolayer Molybdenum Disulphide. An intervalley in-plane spin relaxation channel is revealed due to the opposite effective magnetic fields perpendicular to the monolayer Molybdenum Disulphide plane in the two valleys together with the intervalley electron-phonon scattering. The intervalley electronphonon scattering is always in the weak scattering limit, which leads to a rapid decrease of the inplane spin relaxation time with increasing temperature. A decrease of the in-plane spin relaxation time with the increase of the electron density is also shown.
\end{abstract}

PACS numbers: 72.25.Rb, 81.05.Hd, 71.10.-w, 71.70.Ej

Keywords: Spin relaxation Monolayer $\mathrm{MoS}_{2}$

\section{INTRODUCTION}

Transition-metal dichalcogenides, similar to graphite, are layered materials with weak interlayer van der Waals interaction. These materials can be exfoliated to single to few-layer samples as the fabrication of graphene. $\underline{1}^{-4}$ Among these samples, monolayer Molybdenum Disulphide $\left(\mathrm{MoS}_{2}\right)$ has received much attention due to its distinctive properties,-39 It has a direct gap at the inequivalent $\mathrm{K}$ and $\mathrm{K}^{\prime}$ points, $\stackrel{5,9,20,21,30,33}{=}$ which makes it attractive as a two-dimensional channel material in fieldeffect transistors. Very recently, the field-effect transistor devices based on monolayer $\mathrm{MoS}_{2}$ have been realized in the experiments with high on-off ratio $\underline{4.26} \mathrm{In}$ addition, space inversion symmetry is broken in monolayer $\mathrm{MoS}_{2}$ since it is a two-dimensional hexagonal lattice consisted of two different sublattices, i.e., Mo and $\mathrm{S}$ atoms. The absence of the space inversion symmetry results in a valley-dependent optical selection rule for interband transitions, which allows the realization of the valley polarization by optical pumping with circularly polarized light,$\frac{9,12-14,16,18,22}{2}$ Space inversion symmetry breaking can also induce spin splitting of both the conduction and valence bands where the one of the valence band is much larger than that of the conduction band. $9,18,21,25,29,32,36,37$ This spin splitting is essential for spin physics and spintronic applications. All these intriguing features make monolayer $\mathrm{MoS}_{2}$ of particular interest.

As spin relaxation is crucial for possible realistic spintronic applications, a thorough understanding of the spin relaxation time (SRT) in monolayer $\mathrm{MoS}_{2}$ is essential. Very recently, Ochoa and Roldán 32 investigated the intravalley spin-orbit mediated spin relaxation in monolayer $\mathrm{MoS}_{2}$. For electrons, they calculated the out-of-

*Electric address: mwwu@ustc.edu.cn plane spin relaxation due to the extrinsic Rashba spinorbit coupling (SOC) induced by an external out-of-plane electric field and also the in-plane one due to the intrinsic SOC from the contribution of the valence band due to the space inversion symmetry. However, according to the latest report by Kormányos et al. $\frac{37}{2}$ this intrinsic SOC from the contribution of the valence band is weak since the splitting of the valence band is much smaller than the typical band gap. In contrast, the intrinsic SOC from the conduction band, which manifests itself as a Zeeman-like term with opposite effective magnetic fields in the two valleys, is absent in their calculation. ${ }^{32}$ The Zeeman-like term can give rise to an intervalley spin relaxation channel in the presence of intervalley electron-phonon scattering, which has been shown of significant importance to the spin relaxation in both rippled single-layer graphene $\underline{\underline{40}}$ and also bilayer graphene. $\underline{\underline{41}}$ Moreover, only the disorder is included in a phenomenological manner in their calculation. The electron-electron Coulomb and electron-phonon interaction, which have been demonstrated to play an important role in spin relaxation in semiconductors $\underline{42}$ and also graphene at high temperature, $\stackrel{43}{,}$ are absent.

In the present work, with the electron-electron Coulomb, (both the intra- and inter-valley) electronphonon and the electron-impurity scatterings explicitly included, we study the electron spin relaxation due to the D'yakonov-Perel' 44 mechanism in monolayer $\mathrm{MoS}_{2}$ in the absence of external electric field by the kinetic spin Block equation (KSBE) approach $\stackrel{42}{4}$ According to the latest report by Kormányos et al. $\frac{37}{3}$ the effective magnetic field of the intrinsic SOC of the conduction band is given by

$$
\Omega^{\mu}=2 \omega \mu \hat{\mathbf{z}}
$$

with the $z$-axis being perpendicular to the monolayer $\mathrm{MoS}_{2}$ plane. Here, $\omega$ and $\mu=1(-1)$ represent the strength of the SOC and $\mathrm{K}\left(\mathrm{K}^{\prime}\right)$ valleys, respectively: 37 It is noted that the contribution of the hybridization by the intrinsic SOC of the valence band to the en- 
ergy bands is neglected since the energy scale of this spin splitting is much smaller than the typical band separation $\frac{37}{17}$ This effective magnetic field is momentum independent, indicating an absence of the intravalley inhomogeneous broadening 45 for in-plane spins. Therefore, the intravalley spin relaxation process contributed by the electron-electron Coulomb, intravalley electron-phonon and electron-impurity scatterings are irrelevant to the inplane spin relaxation. Here, only the intervalley process contributes to the in-plane spin relaxation. The underlying physics is the same as the case of bilayer graphene ${ }^{41}$ and also rippled single-layer graphene $\stackrel{40}{\underline{40}}$ This intervalley spin relaxation channel suppresses the in-plane SRT effectively at high temperature. We also find that this system is always in the weak intervalley scattering limit, which determines that the in-plane spin relaxation time is proportional to the intervalley momentum scattering time. As a result, a monotonic decrease of the in-plane SRT with increasing temperature is shown. We also find that the in-plane SRT decreases with the increase of the electron density. Moreover, we show a decrease of the in-plane SRT with increasing initial spin polarization at low temperature, which is very different from the previous studies in both semiconductors $\$ 6,47$ and single-layer graphene ${ }^{43}$ but similar to the case of bilayer graphene. $\underline{41}$

\section{MODEL AND KSBES}

With the effective mass approximation, the Hamiltonian of the conduction band near the $\mathrm{K}\left(\mathrm{K}^{\prime}\right)$ points in monolayer $\mathrm{MoS}_{2}$ can be described by

$$
H_{\mathrm{eff}}^{\mu}=\epsilon_{\mu \mathbf{k}}+\boldsymbol{\Omega}^{\mu} \cdot \boldsymbol{\sigma} / 2,
$$

according to the latest report by Kormányos et al. ${ }^{37}$ Here, $\epsilon_{\mu \mathbf{k}}=\hbar^{2} \mathbf{k}^{2} /\left(2 m^{*}\right)$ with $\mathbf{k}$ and $m^{*}$ being the inplane momentum relative to the $\mathrm{K}\left(\mathrm{K}^{\prime}\right)$ points and the effective mass, respectively. $\boldsymbol{\sigma}$ are the Pauli matrices and $\boldsymbol{\Omega}^{\mu}$ is given in Eq. (1).

We then construct the microscopic KSBEs 42 to investigate the intrinsic electron spin relaxation in monolayer $\mathrm{MoS}_{2}$. The KSBEs are given by $\underline{42}$

$$
\partial_{t} \hat{\rho}_{\mu \mathbf{k}}=\left.\partial_{t} \hat{\rho}_{\mu \mathbf{k}}\right|_{\mathrm{coh}}+\left.\partial_{t} \hat{\rho}_{\mu \mathbf{k}}\right|_{\text {scat }},
$$

in which $\hat{\rho}_{\mu \mathbf{k}}$ stand for the density matrices of electrons with the diagonal terms $\rho_{\mu \mathbf{k}, \sigma \sigma} \equiv f_{\mu \mathbf{k} \sigma}\left(\sigma= \pm \frac{1}{2}\right)$ representing the distribution functions and the off-diagonal ones $\rho_{\mu \mathbf{k},\left(\frac{1}{2}\right)\left(-\frac{1}{2}\right)}=\rho_{\mu \mathbf{k},\left(-\frac{1}{2}\right)\left(\frac{1}{2}\right)}^{*}$ describing the spin coherence. The coherent terms $\left.\partial_{t} \hat{\rho}_{\mu \mathbf{k}}\right|_{\text {coh }}$ are given in Ref. 43. $\left.\partial_{t} \hat{\rho}_{\mu \mathbf{k}}\right|_{\text {scat }}$ are the scattering terms including the electron-electron Coulomb $\left(\left|V_{\mathbf{k}, \mathbf{k}^{\prime}}^{\mu}\right|^{2}\right)$, electronimpurity $\left(\left|U_{\mathbf{k}, \mathbf{k}^{\prime}}^{\mu}\right|^{2}\right)$, intravalley electron-acoustic phonon, electron-optical phonon, and especially the intervalley electron-phonon scattering including the electron-KTA phonon, electron-KLA phonon, electron-KTO phonon and electron-KLO phonon scatterings. Here, KTA, KLA, $\mathrm{KTO}$ and KLO correspond to the transverse acoustic, longitudinal acoustic, transverse optical and longitudinal optical phonon modes at $\mathrm{K}$ point, respectively 20.33 The detailed expressions of the above scattering terms can be found in Ref. 43 (Note that the form factor in the electron-impurity and electron-electron Coulomb scatterings in Ref. 43 is absent here). The scattering matrix elements

$$
\left|V_{\mathbf{k}, \mathbf{k}-\mathbf{q}}^{\mu}\right|^{2}=\left(\frac{V_{\mathbf{q}}^{(0)}}{\varepsilon(\mathbf{q})}\right)^{2}
$$

and

$$
\left|U_{\mathbf{k}, \mathbf{k}-\mathbf{q}}^{\mu}\right|^{2}=Z_{i}^{2}\left|V_{\mathbf{k}, \mathbf{k}-\mathbf{q}}^{\mu}\right|^{2}
$$

with $Z_{i}$ being the impurity charge number (assumed to be 1 in our calculation). The quantity

$$
\varepsilon(\mathbf{q})=1-V_{\mathbf{q}}^{(0)} \sum_{\mu \mathbf{k} s} \frac{f_{\mathbf{k} s}^{\mu}-f_{\mathbf{k}+\mathbf{q} s}^{\mu}}{\epsilon_{\mu \mathbf{k}}-\epsilon_{\mu \mathbf{k}+\mathbf{q}}}
$$

stands for the screening under the random phase approximation. $48.49 V_{\mathbf{q}}^{(0)}=2 \pi e^{2} /(\kappa q)$ is the twodimensional bare Coulomb potential with $\kappa$ being the relative static dielectric constant $\stackrel{21}{=}$ The electron-phonon scattering matrix elements are explicitly given by the latest reports by Kaasbjerg et al. $\stackrel{20,31}{3}$ Specifically, we lay out the matrix elements of the electron-KTA $\left(\left|M_{\mu \mathbf{k}, \mu^{\prime} \mathbf{k}^{\prime}}^{\mathrm{KTA}}\right|^{2}\right)$ and -KLO $\left(\left|M_{\mu \mathbf{k}, \mu^{\prime} \mathbf{k}^{\prime}}^{\mathrm{KLO}}\right|^{2}\right)$ phonon scatterings, which play a dominant role in the in-plane spin relaxation as will be shown later.

$$
\begin{aligned}
\left|M_{\mu \mathbf{k}, \mu^{\prime} \mathbf{k}^{\prime}}^{\mathrm{KTA}}\right|^{2} & =\frac{\hbar^{2}\left(D_{\mathrm{K}, \mathrm{TA}}^{1}\right)^{2} q^{2}}{2 A \rho \Omega_{\mathrm{K}, \mathrm{TA}}} \delta_{\mu^{\prime},-\mu}, \\
\left|M_{\mu \mathbf{k}, \mu^{\prime} \mathbf{k}^{\prime}}^{\mathrm{KLO}}\right|^{2} & =\frac{\hbar^{2}\left(D_{\mathrm{K}, \mathrm{LO}}^{0}\right)^{2}}{2 A \rho \Omega_{\mathrm{K}, \mathrm{LO}}} \delta_{\mu^{\prime},-\mu},
\end{aligned}
$$

where $A$ is the area of the sample; $\rho$ is the mass density of the monolayer $\mathrm{MoS}_{2} ; \Omega_{\mathrm{K}, \mathrm{TA}}$ and $\Omega_{\mathrm{K}, \mathrm{LO}}$ are the energies of KTA and KLO phonon modes, respectively; $D_{\mathrm{K}, \mathrm{TA}}^{1}$ $\left(D_{\mathrm{K}, \mathrm{LO}}^{0}\right)$ is the first- (zeroth-) order deformation potential corresponding to the electron-KTA (-KLO) phonon scattering; $q=\left|\mathbf{k}-\mathbf{k}^{\prime}\right|$. It is noted that the screening by the carriers is included in the electron-phonon scattering in their works with the random phase approximation for acoustic phonon and Debye-Hückel one in the nondegenerate limit for other phonon modes. $\stackrel{48.49}{ }$ It is further noted that they pointed out that the screening can be neglected for the intervalley electron-phonon scattering due to the large wave vectors of phonons $\underline{\underline{20}}$ Therefore, in the present work, we only include the screening for the intravalley electron-phonon scattering with the random phase approximation.

\section{NUMERICAL RESULTS}

In the literature, there still remain controversies over the band structure of monolayer $\mathrm{MoS}_{2}, \frac{5,20,21,25,30,33,36,37}{2}$ 
where different energy gaps, effective masses and the spin splittings are given. Here, we take the effective mass and the strength of the SOC being $m^{*}=0.48 m_{0}{ }^{20.37}$ and $\omega=1.5 \mathrm{meV}, 25,29,37$ respectively. $m_{0}$ stands for the free electron mass. The relative static dielectric constant is chosen to be $\kappa=3.43 \stackrel{21}{2}^{2}$ The mass density $\rho=3.1 \times 10^{-7} \mathrm{~g} / \mathrm{cm}^{2}$; the KTA and KLO phonon energies are $\Omega_{\mathrm{K}, \mathrm{TA}}=23 \mathrm{meV}$ and $\Omega_{\mathrm{K}, \mathrm{LO}}=42 \mathrm{meV}$, respectively; the deformation potentials for the KTA and $\mathrm{KLO}$ phonons are $D_{\mathrm{K}, \mathrm{TA}}^{1}=5.9 \mathrm{eV}$ and $D_{\mathrm{K}, \mathrm{LO}}^{0}=$ $2.6 \times 10^{8} \mathrm{eV} / \mathrm{cm}$, separately ${ }^{20}$ With these parameters, our results are obtained by numerically solving the KS-

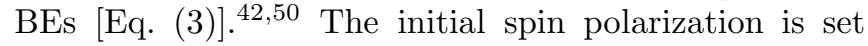
to be $2.5 \%$ and the spin-polarization direction is chosen in the monolayer $\mathrm{MoS}_{2}$ plane unless otherwise specified. As reported, in contrast to the out-of-plane spin orientation, $, 12-14,16,18,22$ the in-plane one is difficult to generate optically due to the large out-of-plane spin splitting of the valence band. However, with an in-plane magnetic field, the in-plane component of the spin polarization can be obtained from the out-of-plane spin orientation ${ }^{51}$ In addition, the in-plane spins can also be realized electrically by ferromagnetic contacts $\stackrel{52.53}{ }$ The electrical method has been widely used in spin transport measurements in semiconductors ${ }^{52}$ and also graphene. ${ }^{53}$

\section{A. Temperature dependence of spin relaxation}

We first study the temperature dependence of the spin relaxation. The in-plane SRT $\tau_{s}$ as function of temperature $T$ is plotted in Fig. 1(a). It is seen that the SRT decreases monotonically with the increase of the temperature. To understand this behavior, we calculate the temperature dependence of the SRT with only the electron-electron Coulomb, electron-impurity, intravalley electron-acoustic phonon, electron-optical phonon, or intervalley electron-phonon scattering included, separately. We find that the contributions of the electron-electron Coulomb, electron-impurity, intravalley electron-phonon scatterings, which only influence the intravalley spin relaxation channel, are negligible due to the absence of a momentum-dependent effective magnetic field of the SOC [Eq. (1)] $]^{42}$ Therefore, the SRT is only determined

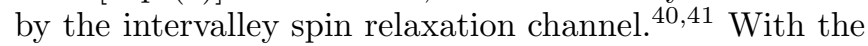
Zeeman-like term being $2 \omega \mu \hat{\mathbf{z}}$ [Eq. (1)], the in-plane SRT is given by

$$
\tau_{s}= \begin{cases}\tau_{v} \quad \text { weak scattering }\left(2 \omega \tau_{v} \geq 1\right) \\ \frac{1}{2 \omega^{2} \tau_{v}} \quad \text { strong scattering }\left(2 \omega \tau_{v} \ll 1\right),\end{cases}
$$

according to the report by Zhang et al $\underline{\underline{40}}$ based on the elastic scattering approximation. Here, $\tau_{v}$ represents the intervalley electron-phonon scattering time. It is noted that the intervalley electron-phonon scattering is always in the weak scattering limit for the electron density upto $2 \times 10^{13} \mathrm{~cm}^{-2}$, therefore $\tau_{s}=\tau_{v}$ throughout our work. As a result, the SRT decreases with the enhancement of the intervalley electron-phonon scattering as the temperature increases.

In addition, we also show a comparison of contributions from two leading intervalley electron-phonon scatterings, i.e., electron-KTA and electron-KLO ones in Fig. 1(a). We find that the electron-KTA phonon scattering plays a more important role in spin relaxation at low temperature whereas the electron-KLO phonon scattering becomes more important at high temperature. This can be understood from the contribution of the electron-phonon scattering matrix element together with phonon number. It is noted that the electron-KTA phonon scattering matrix element is smaller than the electron-KLO phonon one whereas the KTA phonon mode has a larger phonon number due to a smaller phonon energy $\stackrel{20,33}{=}$ At low temperature, the phonon number of KTA mode is much larger than that of KLO mode, which makes the electron-KTA phonon scattering stronger and hence more important to the spin relaxation. However, at high temperature, the phonon numbers of two modes become comparable. Then the electron-KLO phonon scattering plays a more important role in spin relaxation due to the larger scattering matrix element. It is noted that the contributions of the electron-KLA and -KTO phonon scatterings to the spin relaxation are marginal.

\section{B. Electron-density dependence of spin relaxation}

Then we turn to investigate the electron-density dependence of the in-plane SRT and show the results at $T=100$ (300) $\mathrm{K}$ in Fig. 1(b). We find that the SRT decreases rapidly with increasing electron density at $T=100 \mathrm{~K}$ but decreases mildly at $T=300 \mathrm{~K}$. We first focus on the case at $T=100 \mathrm{~K}$. The SRT is dominated by the electron-KTA and -KLO phonon scatterings as pointed out in the temperature dependence of the spin relaxation. The electron-KLO phonon scattering, which has a momentum-independent matrix element [see Eq. [8] ], is insensitive to the electron density. However, the electron-KTA phonon scattering is enhanced with increasing electron density as its matrix element [see Eq. (7)] increases with the increase of the momentum. Therefore, when the electron density increases, the enhancement of the electron-KTA phonon scattering leads to the decrease of the $\operatorname{SRT}\left(\tau_{s}=\tau_{v}\right)$. As for the case at $T=300 \mathrm{~K}$, the increase of the matrix element of the electron-KTA phonon scattering becomes insensitive. In addition, the electron-KLO phonon scattering plays a more important role in spin relaxation than that in the case at $T=100 \mathrm{~K}$. Both lead to a mild decrease of the SRT. 

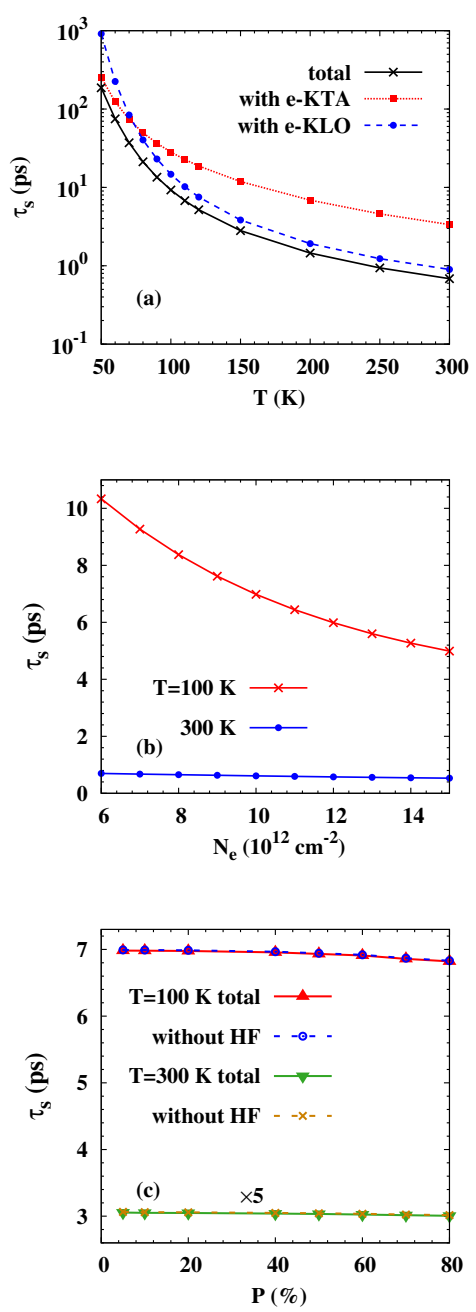

FIG. 1: (Color online) (a) Total in-plane SRT $\tau_{s}(\times)$ and that calculated with only the electron-KTA phonon $(\mathbf{\square})$ or electron-KLO phonon $(\bullet)$ scattering included as function of temperature $T$ with the electron density $N_{e}=7 \times 10^{12} \mathrm{~cm}^{-2}$; (b) $\tau_{s}$ as a function of $N_{e}$ at $T=100(300) \mathrm{K}$; (c) Total inplane SRT $\tau_{s} \boldsymbol{\Delta}(\boldsymbol{\nabla})$ and that calculated without the Coulomb HF term $\circ(\times)$ as function of the initial spin polarization $P$ at $T=100(300) \mathrm{K}$ with $N_{e}=10^{13} \mathrm{~cm}^{-2}$.

\section{Initial spin polarization dependence of spin relaxation}

In the previous investigations in both semiconductor systems ${ }^{46,47}$ and single-layer graphene,, 33 a significant increase of the SRT is shown with the increase of the initial spin polarization, which originates from the contribution of the Coulomb Hartree-Fock (HF) term. However, in the case of bilayer graphene, the SRT decreases rapidly with increasing initial spin polarization at low temperature whereas shows a mild increase at high temperature. ${ }^{41}$ In this case, the SRT is determined by the intervalley electron-phonon scattering. Here, we also investigate the initial spin polarization dependence of spin relaxation. In
Fig.1(c), we plot the in-plane SRT as function of the initial spin polarization $P$ at $T=100(300) \mathrm{K}$. We find that the SRT shows a decrease with increasing $P$ at $T=100 \mathrm{~K}$ whereas becomes insensitive to $P$ at $T=300 \mathrm{~K}$. We first focus on the case of $T=100 \mathrm{~K}$. The decrease of the SRT at $T=100 \mathrm{~K}$ is very different from the previous studies in both semiconductors $\$ 4,47$ and single-layer graphene ${ }^{43}$ but similar to the case of bilayer graphene at low temperature. 41 The underlying physics is understood as follows. The SRT is also determined by the intervalley electron-phonon scattering whereas the contribution of the Coulomb HF term is negligible to the spin relaxation by comparing the calculation with and without the Coulomb HF term shown in Fig. 1(c). The intervalley electron-phonon scattering is in the weak intervalley scattering limit, i.e., the SRT $\tau_{s}=\tau_{v}$ [Eq. (9)] As a result, the SRT decreases with the increase of the initial spin polarization due to the enhancement of the intervalley electron-phonon scattering. ${ }^{41}$ As for the case of $T=300 \mathrm{~K}$, the SRT is also determined by $\tau_{s}=\tau_{v}$ [Eq. (9)]. The insensitive initial spin polarization dependence of $\tau_{s}$ originates from that of $\tau_{v}$ at $T=300 \mathrm{~K} .41$

\section{DISCUSSION AND SUMMARY}

As mentioned previously, the effective magnetic field [see Eq. (10)] is momentum independent, which leads to the absence of the intravalley relaxation channel for inplane spins. This effective magnetic field can become momentum dependent when the contribution of the hybridization by the intrinsic SOC of the valence band to the energy bands is taken into account $\frac{37}{2}$ This intravalley inhomogeneous broadening together with the intravalley scattering opens an intravalley spin relaxation channel. The intravalley process competes with the intervalley one discussed here and the competition between these two processes needs further investigations. ${ }^{32}$ In addition, in real systems, the effective magnetic field can be sensitive to the random local strains ${ }^{54,55}$ due to the symmetry breaking, which also results in an intravalley inhomogeneous broadening and hence the intravalley spin relaxation process. However, this is out of the scope of the present work since the effect of the random local strains on the spin-orbit coupling is still unclear in monolayer $\mathrm{MoS}_{2}$.

In summary, we have investigated the intrinsic electron spin relaxation due to the D'yakonov-Perel' mechanism in monolayer $\mathrm{MoS}_{2}$. The effective magnetic field of the intrinsic SOC is momentum independent, which supplies a Zeeman-like term with opposite effective magnetic fields perpendicular to the mononlayer $\mathrm{MoS}_{2}$ plane in the two valleys. Since it is independent on momentum, the intravalley spin relaxation channel is absent for the in-plane spins. However, the Zeeman-like term, together with the intervalley electron-phonon scattering, gives rise to an intervalley relaxation channel for in-plane spin polarization, which is similar to the case in rippled 
single-layer graphene and also bilayer graphene. This effect has not been yet reported in the literature on monolayer $\mathrm{MoS}_{2}$. The intervalley spin relaxation channel can markedly suppress the in-plane SRT at high temperature. In addition to the intervalley electron-phonon scattering, the electron-impurity, electron-electron Coulomb and intravalley electron-phonon scatterings are also included to calculate the in-plane SRT. However, their contribution to in-plane spin relaxation is negligible due to the absence of intravalley relaxation process. Moreover, we find that the intervalley scattering is always in the weak scattering limit in this material. Therefore, the SRT is always proportional to the intervalley momentum scattering time. This results in a monotonic decrease of the in-plane SRT with the increase of temperature. A decrease of the inplane SRT is also observed in the electron density de- pendence. In addition, we find that the in-plane SRT decreases with the increase of the initial spin polarization at low temperature, which is very different from the previous studies in both semiconductors and single-layer graphene but similar to the case of bilayer graphene.

\section{Acknowledgments}

This work was supported by the National Natural Science Foundation of China under Grant No. 11334014, the National Basic Research Program of China under Grant No. 2012CB922002 and the Strategic Priority Research Program of the Chinese Academy of Sciences under Grant No. XDB01000000.
${ }^{1}$ K. S. Novoselov, D. Jiang, F. Schedin, T. J. Booth, V. V. Khotkevich, S. V. Morozov, and A. K. Geim, PNAS 102, 10451 (2005).

2 A. Ayari, E. Cobas, O. Ogundadegbe, and M. S. Fuhrer, J. Appl. Phys. 101, 014507 (2007).

${ }^{3}$ H. S. S. R. Matte, A. Gomathi, A. K. Manna, D. J. Late, R. Datta, S. K. Pati, and C. N. R. Rao, Angew. Chem. 122, 4153 (2010).

4 B. Radisavljevic, A. Radenovic, J. Brivio, V. Giacometti, and A. Kis, Nature Nanotech. 6, 147 (2011).

5 A. Splendiani, L. Sun, Y. Zhang, T. Li, J. Kim, C.-Y. Chim, G. Galli, and F. Wang, Nano Lett. 10, 1271 (2010).

${ }^{6}$ C. Lee, H. Yan, L. E. Brus, T. F. Heinz, J. Hone, and S. Ryu, ACS Nano 4, 2695 (2010).

7 K. F. Mak, C. Lee, J. Hone, J. Shan, and T. F. Heinz, Phys. Rev. Lett. 105, 136805 (2010).

8 G. Eda, H. Yamaguchi, D. Voiry, T. Fujita, M. Chen, and M. Chhowalla, Nano Lett. 11, 5111 (2011).

9 Z. Y. Zhu, Y. C. Cheng, and U. Schwingenschlögl, Phys. Rev. B 84, 153402 (2011).

10 T. Korn, S. Heydrich, M. Hirmer, J. Schmutzler, and C. Schüller, Appl. Phys. Lett. 99, 102109 (2011).

11 J. K. Ellis, M. J. Lucero, and G. E. Scuseria, Appl. Phys. Lett. 99, 261908 (2011).

12 K. Behnia, Nature Nanotech. 7, 488 (2012).

13 H. Zeng, J. Dai, W. Yao, D. Xiao, and X. Cui, Nature Nanotech. 7, 490 (2012).

14 K. F. Mak, K. He, J. Sahn, and T. F. Heinz, Nature Nanotech. 7, 494 (2012).

15 Q. H. Wang, K. K. Zadeh, A. Kis, J. N. Coleman, and M. S. Strano, Nature Nanotech. 7, 699 (2012).

16 T. Cao, G. Wang, W. Han, H. Ye, C. Zhu, J. Shi, Q. Niu, P. Tan, E. Wang, B. Liu, and J. Feng, Nature Commun. 3, 887 (2012).

17 S. Kim, A. Konar, W.-S. Hwang, J. H. Lee, J. Lee, J. Yang, C. Jung, H. Kim, J.-B. Yoo, J.-Y. Choi, Y. W. Jin, S. Y. Lee, D. Jena, W. Choi, and K. Kim, Nature Commun. 3, 1011 (2012).

18 D. Xiao, G.-B. Liu, W. Feng, X. Xu, and W. Yao, Phys. Rev. Lett. 108, 196802 (2012).

19 W. S. Yun, S. W. Han, S. C. Hong, I. G. Kim, and J. D. Lee, Phys. Rev. B 85, 033305 (2012).
${ }^{20}$ K. Kaasbjerg, K. S. Thygesen, and K. W. Jacobsen, Phys. Rev. B 85, 115317 (2012).

21 T. Cheiwchanchamnangij and W. R. L. Lambrecht, Phys. Rev. B 85, 205302 (2012).

22 G. Sallen, L. Bouet, X. Marie, G. Wang, C. R. Zhu, W. P. Han, Y. Lu, P. H. Tan, T. Amand, B. L. Liu, and B. Urbaszek, Phys. Rev. B 86, 081301(R) (2012).

23 B. D. Kong, C. Zeng, D. K. Gaskill, K. L. Wang, and K. W. Kim, Appl. Phys. Lett. 101, 263112 (2012).

24 D. Lembke and A. Kis, ACS Nano 6, 10070 (2012).

25 E. S. Kadantsev and P. Hawrylak, Solid State Commun. 152, 909 (2012).

${ }^{26}$ H. Liu and P. D. Ye, IEEE Electron Dev. Lett. 33, 546 (2012).

27 M. Buscema, M. Barkelid, V. Zwiller, H. S. van der Zant, G. A. Steele, and A. C. Gomez, Nano Lett. 13, 358 (2013).

28 H.-Z. Lu, W. Yao, D. Xiao, and S.-Q. Shen, Phys. Rev. Lett. 110, 016806 (2013).

29 K. Kośmider and J. F. Rossier, Phys. Rev. B 87, 075451 (2013).

30 H. Shi, H. Pan, Y.-W. Zhang, and B. I. Yakobson, Phys. Rev. B 87, 155304 (2013).

31 K. Kaasbjerg, K. S. Thygesen, and A.-P. Jauho, Phys. Rev. B 87, 235312 (2013).

32 H. Ochoa and R. Roldán, Phys. Rev. B 87, 245421 (2013).

33 X. Li, J. T. Mullen, Z. Jin, K. M. Borysenko, M. B. Nardelli, and K. W. Kim, Phys. Rev. B 87, 115418 (2013).

34 Y. Song and H. Dery, Phys. Rev. Lett. 111, 026601 (2013).

35 H. Rostami, A. G. Moghaddam, and R. Asgari, Phys. Rev. B 88, 085440 (2013).

36 F. Zahid, L. Liu, Y. Zhu, J. Wang, and H. Guo, AIP Advances 3, 052111 (2013).

37 A. Kormányos, V. Zólyomi, N. D. Drummond, P. Rakyta, G. Burkard, and V. I. Fal'ko, Phys. Rev. B 88, 045416 (2013).

38 J. Klinovaja and D. Loss, Phys. Rev. B 88, 075404 (2013).

39 E. Cappelluti, R. Roldán, J.A. S. Guillén, P. Ordejón, and F. Guinea, Phys. Rev. B 88, 075409 (2013).

40 P. Zhang, Y. Zhou, and M. W. Wu, J. Appl. Phys. 112, 073709 (2012).

41 L. Wang and M. W. Wu, Phys. Rev. B 87, 205416 (2013).

${ }^{42}$ M. W. Wu, J. H. Jiang, and M. Q. Weng, Phys. Rep. 493, 
$61(2010)$.

43 Y. Zhou and M. W. Wu, Phys. Rev. B 82, 085304 (2010).

${ }^{44}$ M. I. D'yakonov and V. I. Perel', Zh. Eksp. Teor. Fiz. 60, 1954 (1971) [Sov. Phys. JETP 33, 1053 (1971)]; Fiz. Tverd. Tela (Leningrad) 13, 3581 (1971) [Sov. Phys. Solid State 13, 3023 (1972)]

45 M. W. Wu and C. Z. Ning, Eur. Phys. J. B 18, 373 (2000); M. W. Wu, J. Phys. Soc. Jpn. 70, 2195 (2001).

46 M. Q. Weng and M. W. Wu, Phys. Rev. B 68, 075312 (2003); 70, 195318 (2004).

47 D. Stich, J. Zhou, T. Korn, R. Schulz, D. Schuh, W. Wegscheider, M. W. Wu, and C. Schüller, Phys. Rev. Lett. 98, 176401 (2007); Phys. Rev. B 76, 205301 (2007).

48 G. D. Mahan, Many-Particle Physics (Plenum, New York, 1990).

${ }^{49}$ H. Haug and S. W. Koch, Quantum Theory of the Optical and Electronic Properties of Semiconductors, 4th ed.
(World Scientific, Singapore, 2004).

50 J. M. Kikkawa and D. D. Awschalom, Phys. Rev. Lett. 80, 4313 (1998).

51 D. Stich, J. H. Jiang, T. Korn, R. Schulz, D. Schuh, W. Wegscheider, M. W. Wu, and C. Schüller, Phys. Rev. B 76, 073309 (2007).

52 J. Stephens, J. Berezovsky, J. P. McGuire, L. J. Sham, A. C. Gossard, and D. D. Awschalom, Phys. Rev. Lett. 93, 097602 (2004).

53 N. Tombros, C. Jozsa, M. Popinciuc, H. T. Jonkman, and B. J. van Wees, Nature (London) 448, 571 (2007).

54 R. D. Luttrell, S. Brown, J. Cao, J. L. Musfeldt, R. Rosentsveig, and R. Tenne, Phys. Rev. B 73, 035410 (2006).

55 Q.-C. Sun, X. S. Xu, L. I. Vergara, R. Rosentsveig, and J. L. Musfeldt, Phys. Rev. B 79, 205405 (2009). 